\title{
An Improved DWT-SVD Based Robust Digital Image Watermarking for Color Image
}

\author{
Subin Bajracharya ${ }^{\text {a }}$, Roshan Koju ${ }^{\text {b }}$ \\ ${ }^{a}$ Nepal College of Information Technology, Balkumari, Lalitpur, Nepal \\ ${ }^{b}$ Pulchowk Campus, Lalitpur, Nepal
}

\begin{abstract}
The Digital Watermarking Technique has gained its importance due to its ability to provide the secure mechanism for copyright protection and authenticity of the digital data in this high growing internet and computer technology where the tampering and distribution of digital data illegally from unauthorized users is inevitable. For these two important properties of Digital Watermarking, i.e. Robustness and Imperceptibility of watermarked image must take into consideration. In this paper, invisible robust digital watermarking is proposed using Discrete Wavelet Transform and Singular Value Decomposition in YCbCr Color space. The performance of the proposed algorithm is compared with some previous works and results found are more robust against various geometric attacks.
\end{abstract}

Index Terms: Non-Blind Digital Watermarking, Discrete Wavelet Transform, Singular Value Decomposition, Alpha Blending, Arnold Transformation.

(C) 2017 Published by MECS Publisher. Selection and/or peer review under responsibility of the Research Association of Modern Education and Computer Science.

\section{Introduction}

The rapid growth of internet technology has increased exchange and transmission of digital information. Due to this, it demanded the need of techniques and methods to prevent the tampering and illegal distribution of digital data. One of the techniques used to achieve copyright protection and authenticity of digital data is Digital Watermarking. Digital watermarking is the act of hiding a message related to a digital signal (i.e. An image, song, and video) within the signal itself. Watermarking tries to hide a message related to the actual content of the digital signal [1]. There are several applications of Digital Watermarking such as Broadcast Monitoring, Owner Identification, Proof Ownership, Transaction Tracking, Content Authentication, Copy Control and Device Control [2] [3].

The watermarking techniques fall into two categories. Spatial-Domain methods and Frequency Transform

* Corresponding author. Tel.: 9851129935

E-mail address: subin.bajra@gmail.com 
Domain methods [4] [5]. In Spatial Domain, it directly manipulates digital data to hide the watermark. Its main advantage is low computational complexity. However, this method is vulnerable to different attacks. In frequency domain, digital data to be protected are converted into a frequency domain. Compared to spatial domain methods, it needs more computation, but it can provide better robustness from different attacks [6].

According to distinction, the watermarking system can be classified as Blind, Semi-Blind and Non-Blind [7]. The Blind watermarking scheme is also known as public watermarking scheme. This is the most challenging type of watermarking system as it requires neither the cover (original data), nor the embedded watermark. These systems extract $\mathrm{n}$ bits of the watermark data from the watermarked data (i.e. the watermarked image). The Semi-blind watermarking scheme is also known as semi-private watermarking scheme. This system does not require the cover (original data) for detection. The purpose of this system is to find whether that the watermark can be detected. Non-blind watermarking scheme is also known as private watermarking scheme. This system requires at least the cover (original data) for detection. The System extracts the watermark from the possibly distorted data and uses the original data as a hint.

The paper is organized as follows: Section 2 describes related works, Section 3 described the fundamental concept, in brief, Section 4 describes the proposed Scheme, and Section 5 describes the experimental results. The paper concluded in Section 6 followed by references.

\section{Related Works}

A number of earlier works related to digital image watermarking inspired us to do this research. Some of such recent researches are briefly described in this section.

R. Kaur and H. Singh [13], introduced DWT-DCT-SVD based digital watermarking and implement different attacks on watermarked image such as Gaussian Noise Attack, Salt and Pepper attack and Compression attack. The maximum NC value recorded was 0.995 for Salt and Pepper noise attack. M. Chaudhary, S. Srivastava and V. Chaubey [14], introduced digital watermarking based on DWT-DCT-SVD and Salt and Pepper as an attack on watermarked image. The maximum PSNR value recorded was $19.0941 \mathrm{~dB}$ after the Salt and Pepper attack on the watermarked image. S. R. Hallur, S. Kuri and G. H. Kulkarni [15], proposed a DWT-DCT-SVD based color image digital watermarking in RGB Planes. Different attacks such as AWGN Noise, JPEG 50\% compression, Median filter, Wiener filter, Gaussian Filter, Salt and pepper etc. The the maximum recorded NC value was 1. C. N. Sujatha and P. Satyanarayana [16], proposed DWT-DCT-SVD based color image digital watermarking in RGB planes. Different attacks such as Gaussian Noise, Salt and pepper, Compression, Median Filtering, Average filtering, Sharpening, Rotation etc. to check the robustness of the watermarked Image. The the maximum recorded NC value was 0.9800 for Autumn image for sharpening attack. K. Chaitanya, S. Reddy and G. Rao [17], proposed a color image digital watermarking in RGB planes using DWT-DCT-SVD Coefficients. The the maximum recorded NC value was 0.99 without attack. N. Divecha and D. N. N. Jani [18], proposed a Non-Blind DWT-DCT-SVD based digital watermarking technique for RGBimage. Different attacks like JPEG 50\%, Rotation, Cropping, Gaussian Noise, Gamma Correction, etc. are carried out to check the robustness of the watermarked image. The the maximum recorded NC value was 0.987 for pepper image for Gaussian noise attack. M. A. Rahman and M. M. F. Rabbi [19], proposed a Non-Blind DWT-SVD based watermarking technique for RGB Image. 3-Level DWT is applied on each RGB planes of The cover image and watermark. The SVD is applied to embed the watermark into the cover image. Different attacks like Gaussian, Laplacian, Sobel, Average, Prewitt, Cropping etc. are carried out to check the robustness of the watermarked image. The PSNR value after attacks seems to be at around $54 \mathrm{db}$ to $8 \mathrm{db}$ with different attacks. S. Shekhawat and S. Yadav [20], proposed a NonBlind DWT Based Multiplicative SVD Watermarking Algorithm.1 -Level DWT is applied to the red component of the RGB planes and SVD is applied to embed the watermark into the cover image. J. Guru, H. Dhamecha and B. Patel [21], proposed a Digital Watermarking using a fusion of DWT and SVD. 1 -Level DWT is applied to the red component of the RGB planes and SVD is applied to embed the watermark into the cover image. Different attacks such as Salt and Pepper Noise, Speckle Noise are carried out to check the robustness of the algorithm. 


\section{Concepts}

\subsection{Discrete Wavelet Transform}

Discrete wavelet transformation is based on small waves of limited duration and varying frequency. This is a frequency domain technique in which the firstly the cover image is transformed into the frequency domain and then its frequency coefficients are modified in accordance with the transformed coefficients of the watermark and watermarked image is obtained which is very much more robust. DWT decomposed image hierarchically, providing both spatial and frequency description of the image. It decomposes an image in basically three spatial directions, i.e. horizontal, vertical and diagonal in result separating into four different components, namely LL, LH, HL and HH. Here the first letter refers to applying either low pass frequency operation or high pass frequency operations to the rows and the second refers to the filter applied to the columns of the cover image. LL level is the lowest resolution level, which consists of the approximation part of the cover image and rests three levels, i.e., LH, HL, HH give the detailed information of the cover image.

For the second level of decomposition, any one sub-band is selected and is further decomposed into four levels. The maximum the level of decomposition, the maximum will be the robustness of the watermarked image. At every level of decomposition, the magnitude of DWT coefficients is larger in lower bands (LL), and is smaller in other three bands ( $\mathrm{LH}, \mathrm{HL}$ and $\mathrm{HH}$ ). The larger magnitude of wavelet coefficients shows their higher significance in comparison with the wavelet coefficients of smaller magnitude. Human Visual System is more sensitive to the low frequency parts (the LL sub-band), so the watermark is preferably placed in other three sub-bands to retain the quality of the original image [10].

\subsection{SVD (Singular Value Decomposition)}

Singular Value Decomposition transform is a linear algebra transform which is used for factorization of a real or complex matrix with numerous application in various fields of image processing. As a digital image, can be represented in a matrix form with its entries giving the intensity value of each pixel in the image, SVD of an image $M$ with dimension in mxm is given by.

$$
\mathrm{M}=\mathrm{USV}^{\mathrm{T}}
$$

Where, $\mathrm{U}$ and $\mathrm{V}$ are orthogonal matrices and $\mathrm{S}$ known as singular matrix is a diagonal matrix carrying nonnegative singular values of matrix $\mathrm{M}$. The columns of $\mathrm{U}$ and $\mathrm{V}$ are call left and right singular vectors of $\mathrm{M}$, respectively. They basically specify the geometry details of the original image. Left singular matrix, i.e. $U$ represents the horizontal details and right singular matrix, i.e., V represents the vertical details of the original image. The diagonal value of Matrix $\mathrm{S}$ is arranged in decreasing order which signifies that importance of the entries is decreasing from the first singular value for the last one, this feature is employed in SVD based compression techniques. [10]

There are two main properties of SVD to employ in digital watermarking scheme.

- Small Variation in singular values does not affect the quality of image and

- Singular Values of an image have high stability so; they don't change after various attacks.

\subsection{Alpha Blending}

In Digital Watermarking, the Alpha Blending Technique can be used for embedding watermark into the cover image and extracting watermark from the watermarked image using the below equations, [12]. 
For Watermarking Embedding,

$\mathrm{WMI}=\mathrm{k}^{*}(\mathrm{LL} 1)+\mathrm{q} *(\mathrm{WM} 1)$

For Watermarking Extraction,

$\mathrm{RW}=(\mathrm{WMI}-\mathrm{k} * \mathrm{LL} 1) / \mathrm{q}$

Where, WMI=Watermarked Image, LL1=Frequency Approximation of the Original Image, WM1=Watermark Image, RW=Extracted Watermark Image and k, q are the scaling factors of the original image and watermark respectively.

\subsection{Arnold Transformation}

Arnold transform is used to scramble the watermark image. Arnold transformation is posed in the research of Arnold and the Ergodic theory, which is also called face transformation. For $\mathrm{N} * \mathrm{~N}$ image, Arnold transform is defined as

$$
\left(\begin{array}{l}
x^{\prime} \\
y^{\prime}
\end{array}\right)=\left(\begin{array}{ll}
1 & 1 \\
1 & 2
\end{array}\right)\left(\begin{array}{l}
x \\
y
\end{array}\right) \bmod (N)
$$

Where $\mathrm{x}, \mathrm{y}$ are the coordinates of the watermark image and $\mathrm{x}$ ', $\mathrm{y}^{\prime}$ are the coordinates after scrambling and $\mathrm{N}$ is the size of the watermark image. The time of restoring a watermark image after scrambling by using Arnold transform is rising with increasing the image size [6].

\subsection{YCbCr Color Space}

YCbCr is a family of color space used as a part of the color image pipeline in video and photography system. $\mathrm{Y}$ is the luma component that shows the brightness and $\mathrm{Cb}$ and $\mathrm{Cr}$ are blue difference and red difference Chroma components. YCbCr is not an absolute color space; rather, it is a way of encoding RGB information. $\mathrm{RGB}$ to $\mathrm{YCbCr}$ conversion can be obtained from the following equations

$$
\begin{aligned}
& Y=0.299 R+0.587 G+0.114 B \\
& C b=0.596 R-0.272 G-0.321 B \\
& C r=0.212 R-0.532 G-0.311 B
\end{aligned}
$$

\section{Proposed Algorithm}

The algorithm proposed here is a Non-Blind Digital Watermarking where there is a need of the cover image for the watermark extraction.

\subsection{Watermark Embedding}

1. Read RGB Color The cover image

2. Convert RGB Color Image into $\mathrm{YCbCr}$ each color space

3. Select by Color channel and apply 4-level DWT to subdivide the image into LL4, LH4, HL4 and HH4

4. Select HH4 sub-band and apply SVD to that sub band, resulting CU, CS, CV 

5. Read Color watermark image
6. Extract R Channel from RGB Color Watermark
7. Apply Arnold Transformation to Scramble the R Color Channel.
8. Apply 3-level DWT on the scrambled R Channel to divide the image into WLL3, WLH3, WHL3 and WHH3
9. Select WHH3 sub band and apply SVD to that sub band, resulting WU, WS, WV
10. Embed watermark to the cover image using following alpha blending equation
11. $\mathrm{SNEW}=\mathrm{CS} * \mathrm{k}+\mathrm{q} * \mathrm{WS}$ where $\mathrm{k}$ and $\mathrm{q}$ are scaling factor
12. $\mathrm{S}=\mathrm{CU} * \mathrm{SNEW} * \mathrm{CV}$,
13. Apply inverse 4-Level DWT on S to obtain Y Channel Watermarked Image
14. Combine $\mathrm{Y}, \mathrm{Cb}$ and $\mathrm{Cr}$ color channels to form RGB Color Space watermarked image

\subsection{Watermark Extraction}

1. Read The cover image and Watermarked Image

2. Convert The cover image and Watermarked Image From RGB Color Space to YCbCr Color Space

3. Apply 4-Level DWT on Y Channel of The cover image to sub divide the cover image into HLL4,HLH4,HHL4 and HHH4

4. Apply 4-Level DWT on Y Channel of Watermarked Image to subdivide the Watermarked Image into WMLL4, WMLH4, WMHL4 and WMHH4

5. Read Color Watermark Image

6. Extract R,G and B Channel from RGB Color watermarkImage

7. Apply Arnold Transformation to Scramble the R Color Channel.

8. Apply 3 level DWT on scrambled R channel to divide the it into WLL3, WLH3, WHL3 and WHH3

9. Select WHH3 sub band and apply SVD to that sub band, resulting WU, WS, WV

10. Select a HHH4 sub - band and apply SVD to that sub band, resulting CU, CS, CV

11. Select WMHH3 sub-band and apply SVD to that sub band, resulting WMU, WMS, WMV

12. Extract watermark using following alpha blending equation

13. $\mathrm{SNEW}=\left(\mathrm{WMS}-\mathrm{q}^{*} \mathrm{CS}\right) / \mathrm{k}$ where $\mathrm{k}$ and $\mathrm{q}$ are scaling factor

14. $\mathrm{S}=\mathrm{WU} * \mathrm{SNEW}^{*} \mathrm{WV}$,

15. Apply Inverse 3-Level DWT on S

16. Apply Anti-Arnold Transformation to unscramble the image extracted from Step15

17. Combine R, G and B Color Channel to get the RGB Color Watermark

\section{Experiments and Results}

In this section, experimental results of the proposed method are presented. The algorithm is realized in C\#. The performance is evaluated using Peak Signal to Noise Ratio (PSNR) and Normalized Cross-Correlation (NC). The Standard RGB color images (Lena and Pepper) of size 512x512 are used as the cover image and RGB color image of size 256x256 is used as the watermark image. The cover images and watermark image are shown in figure 1. Two major tests, imperceptibility and robustness are carried out with varying values of scaling factor $\mathrm{k}$ and $\mathrm{q}$ and results are compared with other existing watermark algorithms. 


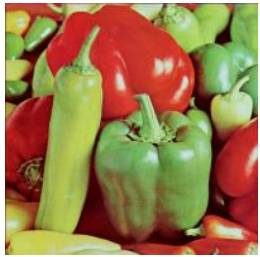

(a)

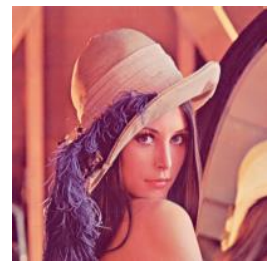

(b)

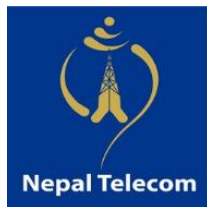

(c)

Fig.1. Standard Test and Watermark Image (a) Pepper (b) Lena (c) Watermark

\subsection{Imperceptibility Test}

Imperceptibility is measure of transparency and is measured via performance measures like a peak signal to noise ratio (PSNR) and Mean Square Error (MSE). The peak signal to noise ratio (PSNR) is typically used as a measure for the imperceptibility of a watermarked image using performance measures. If $\mathrm{W}$ is the original image and $\mathrm{W}^{\prime}$ is modified image, then PSNR is calculated as

$$
P S N R_{d B}=20 \times \log _{10}\left(\frac{M A X}{\sqrt{M S E}}\right)
$$

Where MSE $=$ Mean square Error is given by

$$
M S E=\frac{1}{m \times n} \sum_{k=n}^{m} \sum_{i=1}^{n}\left(f(k, l)=f^{\prime}(k, l)\right)^{2}
$$

Where, $f(k, l)$ is host image and $f(k, l)$ is watermarked image to determine the optimum value of $k$ and $q$, both are varied from 0.01 to 0.90 and best value of PSNR, MSE and NC are recorded at $k=0.3$ and $q=0$. 9. The Table 1 shows the recorded value of PSNR for Lena and Pepper.

Table 1. PSNR and MSE (The cover image vs. Watermarked Image) for Pepper and Lena with $\mathrm{q}=0.3$ and $\mathrm{k}=0.9$

\begin{tabular}{cccc}
\hline Image & MSE & PSNR $(\mathrm{dB})$ & NC \\
\hline Peppers & 0.98828 & 48.1819 & 0.9999 \\
Lena & 1.3015 & 47.9971 & 0.9999 \\
\hline
\end{tabular}

Figure 2 below shows the watermarked Pepper image and Lena image

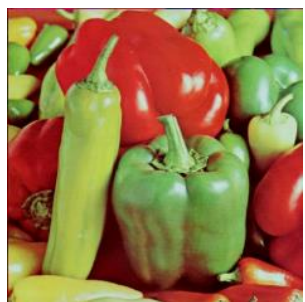

a)

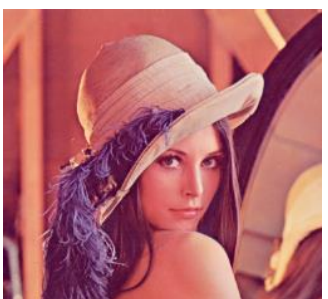

(b)

Fig.2. Watermarked Image (a) Pepper (b) Lena 


\subsection{Robustness Test}

To test the robustness of the proposed algorithm, different geometric attacks were performed on the watermarked image and watermark was extracted. Robustness of image was tested by comparing the similarity of extracting a watermark image with the original watermark image. Similarity of watermarks were carried out on the basis of normalized cross correlation (NC).

$\mathrm{NC}$ is expressed as follows

$$
N C=\frac{\sum \sum\left[W(u, v) W^{\prime}(u, v)\right]}{\sqrt{\sum \sum W(u, v)^{2} \sqrt{\sum \sum W^{\prime}(u, v)^{2}}}}
$$

The Table below shows the NC value between the original watermark and extracted watermark at $\mathrm{k}=0.3$ and $\mathrm{q}=0.9$

Table 2. NC (Original watermark Image vs. Extracted watermark) for Pepper and Lena with q=0.3 and k=0.9

\begin{tabular}{ccc}
\hline Attacks & Pepper & Lena \\
\hline JPEG Compression 10\% & 0.9998 & 0.9999 \\
JPEG Compression 50\% & 0.9999 & 0.9999 \\
Rotation 5 Degree & 0.9999 & 0.9999 \\
Rotation 45 Degree & 0.9999 & 0.9999 \\
Rotation 90 Degree & 1 & 1 \\
Normal Blur & 0.9998 & 0.9999 \\
Gaussian Blur & 0.9998 & 0.9998 \\
Motion Blur & 0.9998 & 0.9999 \\
Salt and Pepper (80) & 0.9996 & 0.9996 \\
Gaussian Noise & 1 & 1 \\
Contrast Stretching & 1 & 1 \\
Sharpening & 0.997 & 0.9972 \\
Histogram Equalization & 0.9998 & 0.9999 \\
Gray Scale & 1 & 1 \\
Median Filter & 0.9999 & 0.9999 \\
Mean Filter & 0.9998 & 0.9999 \\
Top Cropping & 1 & 1 \\
Bottom Cropping & 1 & 1 \\
Left Cropping & 1 & 1 \\
Right Cropping & 1 & 1 \\
Center Cropping & 1 & 1 \\
\hline & & \\
\hline & 1 & 1 \\
\hline
\end{tabular}

The recorded NC values are close to 1 which depicts high similarity between the original watermark and extracted watermark after different geometric attacks. The different geometric attacked watermarked pepper image is shown in figure 3 . 


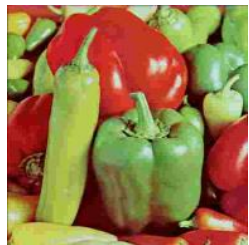

JPEG Compression $10 \%$

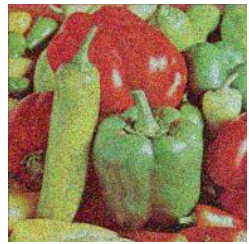

Salt and Pepper

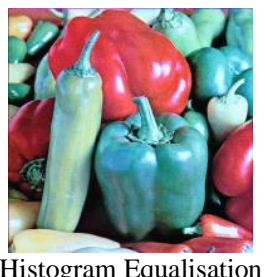

Histogram Equalisation

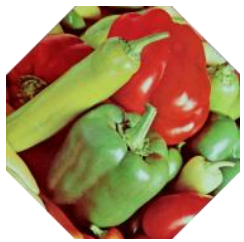

Rotation 45 Degree

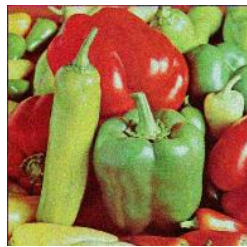

Contrast Stretching

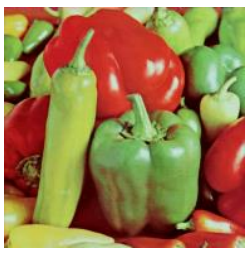

Median Filter

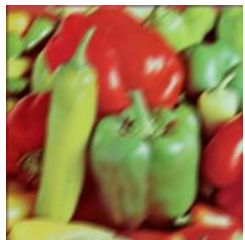

Motion Blur

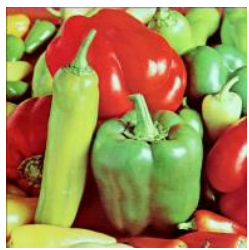

Sharpening

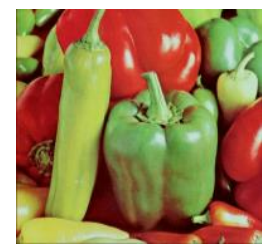

Top Cropping

Fig.3. Geometric attacked Pepper Watermarked Images

\subsection{Comparison with existing Algorithms}

For Comparison, the performance measures recorded for proposed algorithm were compared with performance measures of 3 other existing algorithms. The same cover image and watermark that was used by comparing algorithm was used to compare the performance measures.

Table 3. Comparison of Robustness between Proposed Algorithm and Referenced Algorithm

\begin{tabular}{ccc}
\hline Attacks/Image & $\begin{array}{c}\text { Referenced } \\
\text { Algorithm [16] }\end{array}$ & Proposed Algorithm \\
Autumn & NC & NC \\
\hline Gaussian Noise & 0.9797133 & 0.999962 \\
Salt and Pepper (80) & 0.9799189 & 0.999464 \\
JPEG Compression & 0.9795032 & 0.999943 \\
Median Filter & 0.9795671 & 0.999965 \\
Average Filter & 0.9795031 & 0.999948 \\
Sharpening & 0.9800366 & 0.998923 \\
Rotation & 0.9795475 & 0.999968 \\
Histogram Equalization & 0.9798538 & 0.999929 \\
Right Cropping & 0.9766723 & 0.999997 \\
Left Cropping & 0.9763842 & 0.999997 \\
Top Cropping & 0.9732001 & 0.999997 \\
Bottom Cropping & 0.9730237 & 0.999997 \\
Motion Blur & 0.9794848 & 0.99995 \\
\hline
\end{tabular}


The NC value of the proposed algorithm observed to be better for geometric attacks listed in table 3 than that of reference algorithm.

Table 4. Comparison of Robustness between Proposed Algorithm and Referenced Algorithm

\begin{tabular}{|c|c|c|}
\hline $\begin{array}{c}\text { Attacks/Image } \\
\text { Pepper } \\
\end{array}$ & $\begin{array}{c}\text { Referenced } \\
\text { Algorithm [15] } \\
\text { NC } \\
\end{array}$ & $\begin{array}{c}\text { Proposed Algorithm } \\
\text { NC } \\
\end{array}$ \\
\hline AWGN Noise & 0.9983 & 0.99998 \\
\hline JPEG Compression $50 \%$ & 0.9999 & 0.99996 \\
\hline Median Filter & 0.9999 & 0.99994 \\
\hline Gaussian Filter & 1 & 0.99991 \\
\hline Salt and Pepper & 0.999 & 0.99993 \\
\hline Sharpen Image & 1 & 0.99822 \\
\hline Image Rotation 45 & 0.9997 & 0.99996 \\
\hline Image Rotation 90 & 0.9999 & 1 \\
\hline
\end{tabular}

The NC value of the proposed algorithm observed to be better for geometric attacks listed in table 4 than that of reference algorithm.

Table 5. Comparison of Robustness between Proposed Algorithm and Referenced Algorithm

\begin{tabular}{ccc}
\hline Attacks/Image & $\begin{array}{c}\text { Referenced } \\
\text { Algorithm [18] } \\
\text { NC }\end{array}$ & $\begin{array}{c}\text { Proposed Algorithm } \\
\text { Peppers }\end{array}$ \\
JPEG 20\% & 0.9995 & NC \\
Crop & 0.9992 & 0.999839 \\
Rotation 45 & 0.9988 & 0.999996 \\
Gamma Correction 0.8 & 0.999 & 0.999901 \\
Gamma Correction 1.2 & 0.9991 & 0.999919 \\
Gaussian Noise & 0.985 & 0.999911 \\
Contrast Adjust & 0.9991 & 0.999989 \\
Salt and Pepper & 0.994 & 0.999994 \\
Sharpen & 0.9952 & 0.999594 \\
& & 0.996976 \\
\hline
\end{tabular}

The NC value of the proposed algorithm observed to be better for geometric attacks listed in table 5 than that of reference algorithm.

The proposed algorithm seems to be very robust for different geometric attacks as compared to the other existing watermarking algorithm because of the following reasons. The YCbCr Color Space is used instead of RGB Color space as, RGB Color Channel is complex in describing the color pattern and has redundant information between each component and are highly correlated. Also, according to [11], embedding watermarks in the color channels of $\mathrm{YCbCr}$ are more robust and imperceptible than RGB Color Channels. The Arnold Transformation is used as an image scrambling technique to scramble the watermark image. The image scrambling technique is used as the pre-processing or post-processing, to transform a meaningful image into meaningless or disordered image in order to enhance the power to resist attacks and in turn increase the robustness [6]. For embedding watermark image in the cover image and extraction watermark from watermarked image, Alpha blending technique is being used. In this technique, two scaling parameters are opted for watermarking. With, Alpha Blending has two scaling factors, we can vary scaling factors for both watermark image and the original image to get better results for embedding and extraction of the watermark [12]. In this paper, 4 Level DWT is carried out for the cover image and 3 Level DWT for watermark image.The higher the DWT Level, the higher will be the robustness of the watermarked image [10]. 


\section{Conclusion}

In this paper, a DWT-SVD based digital watermarking was proposed using Alpha Blending and Arnold Transformation in YCbCr Color space. The proposed method is the Non-Blind Digital Watermarking where there is a need of the cover image for the watermark extraction. With the good value of PSNR that defines the imperceptibility, we can say that the cover image and watermarked image are visually same. And with high value of $\mathrm{NC}$ that defines the robustness, after different geometric attacks, shows the high similarity between the original watermark and extracted watermark. These readings show that the proposed algorithm seems to behave more robustness against different geometric attacks in comparison to referenced algorithms.

\section{References}

[1] M. Durvey and D. Satyarthi, "A Review Paper on Digital Watermarking," International Journal of Emerging Trends \& Technology in Computer Science (IJETTCS), vol. 3, no. 4, pp. 99-105, 2014.

[2] Cox, M. L. Miller and J. A. Bloom, "Digital Watermarking," Morgan Kaufmann Publishers, 2002.

[3] R. G. Schyndel, A. Z. Tirkel and C. F. Osborne, "A Digital Watermark," in Proceedings of 1994 International Conference, Austin, Texas, 1994.

[4] H. Daren, L. J. H. Jiwu and L. Hongmei, "A DWT Based Image Watermarking Algorithm," in Proceedings of the IEEE International Conference on Multimedia and Expo, 2001.

[5] M. Barni, F. Bartolini, V. CappeAini and A. Piva, "A DCT-Domain System for Robust Image Watermarking," Vols. 66, No.3, pp. 357-372, 1998.

[6] S. Madhesiya and S. Ahmed, "Advanced Technique of Digital Watermarking based on SVD-DWT-DCT and Arnold Transform," International Journal of Advanced Research in Computer Engineering \& Technology (IJARCET), Vols. 2, No 5, May 2013.

[7] H. Jahankhani, D. L. Watson, G. Me and F. Leonhardt, "Authentication," in Handbook of Electronic Security and Digital Forensics, Singapore, World Scientific Publishing Co. Pte. Ltd, 2010, p. 40.

[8] S. Kashyap, "Digital Watermarking Techniques and Various Attacks Study for Copyright Protection," International Journal of Advanced Research in Comuter Science and Software Engineering, vol. 5, no. 3, pp. 737-745, 2015.

[9] Akter and M. A. Ullah, "DIGITAL WATERMARKING WITH A NEW ALGORITHM," International Journal of Research in Engineering and Technology (IJRET), vol. 3, no. 3, 2014.

[10] N. Bisla and P. Chaudhary, "Comparative Study of DWT and DWT-SVD Image Watermarking Techniques," International Journal of Advanced Research in Computer Science and Software Engineering, vol. 3, no. 6, pp. 821-825, 2013.

[11] R. Koju and S. R. Joshi, "Comparative Analysis of Color Image Watermarking Technique in RGB,YUV and YCbCr Color Channels," Nepal Journal of Science and Technology, vol. 15, pp. 133-140, 2014.

[12] P. Sharma and S. Swami, "Digital Image Watermarking Using 3 level Discrete Wavelet Transform," Conference on Advances in Communication and Control Systems 2013 (CAC2S 2013), 2013.

[13] R. Kaur and H. Singh, "An Improved Performance of Watermarking In DWT Domain Using SVD," International Journal of Latest Trends in Engineering and Technology (IJLTET), vol. 5, no. 1, pp. 459465, March 2015.

[14] M. Chaudhary, S. Srivastava and V. Chaubey, "DWT-DCT-SVD Based Digital Image Watermarking Using Salt and Pepper Method," International Journal of Advance Research In Science And Engineering, vol. 4, no. 6, pp. 34-38, June 2015.

[15] S. R. Hallur, S. Kuri and G. H. Kulkarni, "Robust Digital Watermarking using DWT-DCT-SVD Algorithms for Color Image," International Journal of Current Engineering and Technology, vol. 5, no. 4, pp. 2722-2726, August 2015. 
[16] C. N. Sujatha and P. Satyanarayana, "An Improved Hybrid Color Image Watermarking under Various Attacks," International Journal of Advanced Research in Computer and Communication Engineering, vol. 4, no. 3, pp. 339-343, March 2015.

[17] K. Chaitanya, S. Reddy and G. Rao, "Digital Color Image Watermarking In RGB Planes Using DWTDCT-SVD Coefficients," International Journal of Computer Science and Information Technologies (IJCSIT), vol. 5, no. 2, pp. 2413-2417, 2014.

[18] N. Divecha and D. N. N. Jani, "Implementation and performance analysis of DCT-DWT-SVD based watermarking algorithms for color images," International Conference on Intelligent Systems and Signal Processing (ISSP), pp. 204-208, 2013.

[19] M. A. Rahman and M. M. F. Rabbi, "Non-Blind DWT-SVD based Watermarking Technique for RGB Image," Global Journal of Researches in Engineering: Electrical and Electronics Engineering, vol. 15, no. 4, 2015.

[20] S. Shekhawat and S. Yadav, "NonBlind DWT Based Multiplicative SVD Watermarking Algorithm," International Journal of Emerging Technology and Advanced Engineering, vol. 4, no. 1, pp. 289-293, 2014.

[21] J. Guru, H. Dhamecha and B. Patel, "Fusion of DWT and SVD digital watermarking Techniques for robustness," International Journal of Advanced Research in Computer Science and Software Engineering, vol. 4, no. 9, pp. 791-797, 2014.

\section{Author's Profiles}

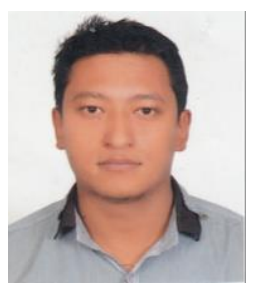

Subin Bajracharya: Graduated master's degree in Computer Engineering from Nepal College of Information Technology, Lalitpur, Kathmandu Nepal, 2016. His research areas include Image Processing, Networking, Cloud Computing, Big Data, Wireless Communication.

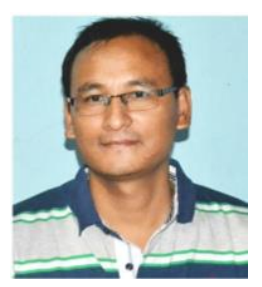

Roshan Koju: PHD Research Student in Pulchowk Campus, IOE, TU, Lalitpur, Nepal. He has graduated master's degree in Computer Engineering from Nepal College of Information Technology, Lalitpur, Kathmandu Nepal, 2011 His research areas include Image processing, Networking, Wireless Communication.

How to cite this paper: Subin Bajracharya, Roshan Koju,"An Improved DWT-SVD Based Robust Digital Image Watermarking for Color Image", International Journal of Engineering and Manufacturing(IJEM), Vol.7, No.1, pp.49-59, 2017.DOI: 10.5815/ijem.2017.01.05 